\title{
Volatile oil from Saussurea lappa exerts antitumor efficacy by inhibiting epithelial growth factor receptor tyrosine kinase- mediated signaling pathway in hepatocellular carcinoma
}

\author{
Xuejing Lin ${ }^{1, *}$, Zhangxiao Peng ${ }^{1, *}$, Xiaohui Fu ${ }^{2,}{ }^{*}$, Chunying Liu ${ }^{1}$, Yang Xu${ }^{1}$, Weidan $\mathrm{Ji}^{1}$, \\ Jianhui Fan'1, Lei Chen", Lin Fang ${ }^{3}$, Yao Huang ${ }^{2}$, Changqing Su ${ }^{1,3}$ \\ ${ }^{1}$ Department of Molecular Oncology, Eastern Hepatobiliary Surgical Hospital \& National Center of Liver Cancer, Second \\ Military Medical University, Shanghai 200438, China \\ ${ }^{2}$ Department of Biliary Tract Surgery, Eastern Hepatobiliary Surgical Hospital, Second Military Medical University, Shanghai \\ 200438, China \\ 3 Jiangsu Center for The Collaboration and Innovation of Cancer Biotherapy, Xuzhou Medical College, Xuzhou 221002, China \\ *These authors have contributed equally to this work \\ Correspondence to: Changqing Su, email: suchangqing@gmail.com \\ Yao Huang, email: huangyaodr@msn.com
}

Keywords: VOSL, hepatocellular carcinoma, epithelial growth factor receptor, signaling, xenograft model

Received: June 20, 2016

Accepted: October 19, 2016

Published: October 28, 2016

\section{ABSTRACT}

Hepatocellular carcinoma (HCC) treatment remains lack of effective chemotherapeutic drugs, therefore, discovering novel anti-HCC drugs is a very attractive and urgent task. In this study, we reported VOSL (volatile oil from Saussurea lappa root) exhibits potent therapeutic effect on SMMC-7721 xenografts without obvious side effects. In the in vitro experiments, VOSL inhibited HCC cell proliferation by arresting cell cycle at $S$ and $\mathrm{G2} / \mathrm{M}$ phases, and induced HCC cell apoptosis by activating the Caspase 3 pathway. VOSL also decreased the capability of HCC cell migration and invasion through MMP-9 depression. Moreover, mechanistic study indicated that VOSL can act as an epithelial growth factor receptor (EGFR) inhibitor to suppress EGFR activation and then to suppress its downstream MEK/P38 and PI3-K/Akt pathways. These results suggested that VOSL may be a novel antiHCC drug candidate.

\section{INTRODUCTION}

Hepatocellular carcinoma (HCC) is a most malignant cancer which generally arises from various kinds of chronic liver diseases [1-3]. The five year survival rate of HCC patients after surgery is only about $20 \%-30 \%$. Frequent recurrence, metastasis and multi-drug resistance are the main reasons for the high mortality rate of HCC $[4,5]$. Up to now, sorafenib is the only effective target chemotherapeutic agent for late-stage HCC patients, however, its therapeutic effect is still rather disappointing. Therefore, to discovernovel drugs is an urgent work for the treatment of HCC.

During a long time, natural products have been served as important sources of novel lead structures for discovery of anticancer agents as their diverse 'drug-like' structure and 'biologically friendly' molecular qualities [6-9]. According to a recent analysis, at least 73 clinically approved anticancer drugs are plant-derived agents, including some well-known chemotherapeutic agents, such as vinblastine, etoposide, paclitaxel, topotecan $[8,10]$.

The root of Saussurea lappa, has been used in the treatment of cancer for thousands of years in China. It includes a variety of bioactive ingredients like volatile oil, sterols, alkaloids, organic acids and so on. Our previous studies demonstrated that the volatile oil from Saussurea lappa root (VOSL), sesquiterpene lactones-rich fraction, contains the most important anti-cancer constituents [9]. Thereinto, costunolide (Cos) and dehydrocostuslactone (Dehy) are the main natural sesquiterpene lactones in VOSL, account for about $75 \%$ by weight. Numerous researches reported that these two compounds exhibited potential anti-cancer activities towards various types of cancer, including leukemia, bladder cancer, breast cancer, prostatic cancer, and so on [10]. Cos and Dehy have anti- 
hepatitis B virus activity, which is very important for prevention of liver cancer [11]. Therefore, we thought that VOSL and its main bioactive constituents, Cos and Dehy, may be potential drug candidates for prevention and treatment of HCC.

Our previous study also revealed that Cos and Dehy exhibited synergistic anti-cancer effect in vivo. VOSL showed stronger inhibition effects on human breast cancer MCF-7 xenografts than Cos or Dehy alone. Moreover, VOSL not only inhibited the growth of tumors, but also maintained weight and vitality of tumor-burdened nude mice, which is a great significance to "tumor-burdened survival" [12]. However, the synergistic anti-tumor mechanism of VOSL remains vague. Therefore, we investigated the anti-HCC efficiency and molecular mechanisms of VOSL in order to promote its pre-clinical research, which may offer a novel chemotherapeutic agent for HCC prevention and treatment.

\section{RESULTS}

\section{VOSL suppresses HCC cell proliferation and HCC xenograft growth}

VOSL, Cos and Dehy were obtained from the dry roots of Saussurea lappa through a bioactivity-oriented screening platform in previous study (Figure 1A) [9]. MTT assay showed that SMMC-7721 and Hep3B cells were more sensitive to these compounds. Simultaneously, the inhibitory rate of VOSL on normal liver cells, especially WRL-68 cells, was the lowest, which revealed the relatively weak toxicity on normal cells (Figure 1B).

SMMC-7721 xenografts in nude mice were used to evaluate the anti-HCC effects of VOSL and its active ingredients, and the results demonstrated that VOSL revealed the best anti-HCC activity among five test groups (Figure 1C, 1D and 1E). The inhibitory rates of VOSL, 5-Fu, C+2D, Dehy, and Cos on SMMC-7721 xenografts are $55.71 \%, 42.22 \%, 37.51 \%, 30.23 \%$, and $23.15 \%$, respectively, after intraperitoneal injections for 24 times.

\section{VOSL inhibits clone formation of $\mathrm{HCC}$ and modulates cell cycle progression}

To investigate the long-term cytotoxicity of VOSL and its active ingredients on HCC cells, cell clone formation assay was carried out on SMMC-7721 and Hep3B cells. The results revealed that VOSL, C $+2 \mathrm{D}$, Cos and Dehy dose-dependently inhibited the clone formation of SMMC-7721 and Hep3B cells (Figure 2A and 2B; Supplementary Figure S1). The uncontrolled cell cycle progression is one of the hallmarks of cancer, therefore, agents targeting this process may be potential chemopreventive drugs [10]. Our results demonstrated that VOSL, C $+2 \mathrm{D}, \mathrm{Cos}$, and Dehy dose-dependently modulated SMMC-7721 cell cycle progression through $\mathrm{S}$ phase increase combined with G0/G1 depletion. Moreover, Cos changed Hep3B cell cycle progression by increasing $\mathrm{S}$ phase and decreasing G0/G1 and G2/M phases. However, VOSL, C+2D and Dehy changed Hep3B cell cycle progression by increasing $\mathrm{S}$ and $\mathrm{G} 2 / \mathrm{M}$ phases combined with decreasing G0/G1 phase (Figure 2C and Table 1).

\section{VOSL induces HCC cell apoptosis}

Hoechst analysis revealed that cell nuclei of HCC cells in the tested groups experienced a stronger blue fluorescence than those in the control group, which meant that VOSL, C+2D, Cos, and Dehy treatment can induce apoptosis of SMMC-7721 and Hep3B cells accompanied with nucleus condensation (Figure 3A).

Annexin V-FITC/PI staining showed that VOSL, $\mathrm{C}+2 \mathrm{D}, \mathrm{Cos}$, and Dehy treatments led to a dramatic increase of apoptotic ratio in both SMMC-7721 and Hep3B cells (Figure 3B and 3C; Supplementary Figure S2). Active Caspase 3 is the primary markers of cells undergo apoptosis. Cleaved-Caspase 3 and Caspase 3 were measured by Western blot at $48 \mathrm{~h}$ later after treatment. The results showed VOSL, C+2D and Dehy treatments up-regulated the expression of cleaved-Caspase 3 and down-regulated the expression of Caspase3 in SMMC-7721 and Hep3B cells. However, the expression of Caspase 3 and cleavedCaspase 3 in the Cos-treated group had no obvious changes compared with the control group, which meant that the apoptotic induction mechanism of Cos on HCC cells may be different from VOSL, C+2D and Dehy (Figure 3D).

\section{VOSL inhibits the capbility of HCC cell motility}

The majority of HCC patients die of tumor recurrence and metastasis, therefore, inhibiting the capbility of HCC cell motility is an attractive strategy for its treatment [10]. We examined the possible effects of VOSL, C $+2 \mathrm{D}, \mathrm{Cos}$, and Dehy on HCC cell migration and invasion through wound healing and Transwell assay. The results indicated that VOSL, C+2D, Cos, and Dehy, at low concentrations that did not notably reduce the viability of HCC cells, dose-dependently reduced the capabilities of HCC cell migration and invasion when compared with the untreated group (Figure 4A-4D; Supplementary Figure S3 and S4). Moreover, MMP-9, a major member of matrix metalloproteinases (MMPs), was measured through Western blot, the results showed that its expression was significantly reduced in both SMMC-7721 and Hep3B cells after treatment (Figure 4E).

\section{VOSL inhibits EGFR activation in HCC cells}

Activation of the extracellular signal-regulated kinases (ERK), the Jun N-terminal kinases (JNK) and the p38 MAPKs plays an important part in regulating of cancer cell biological characteristics $[14,15]$. PI3-K/ Akt signaling is widely involved in controlling the 


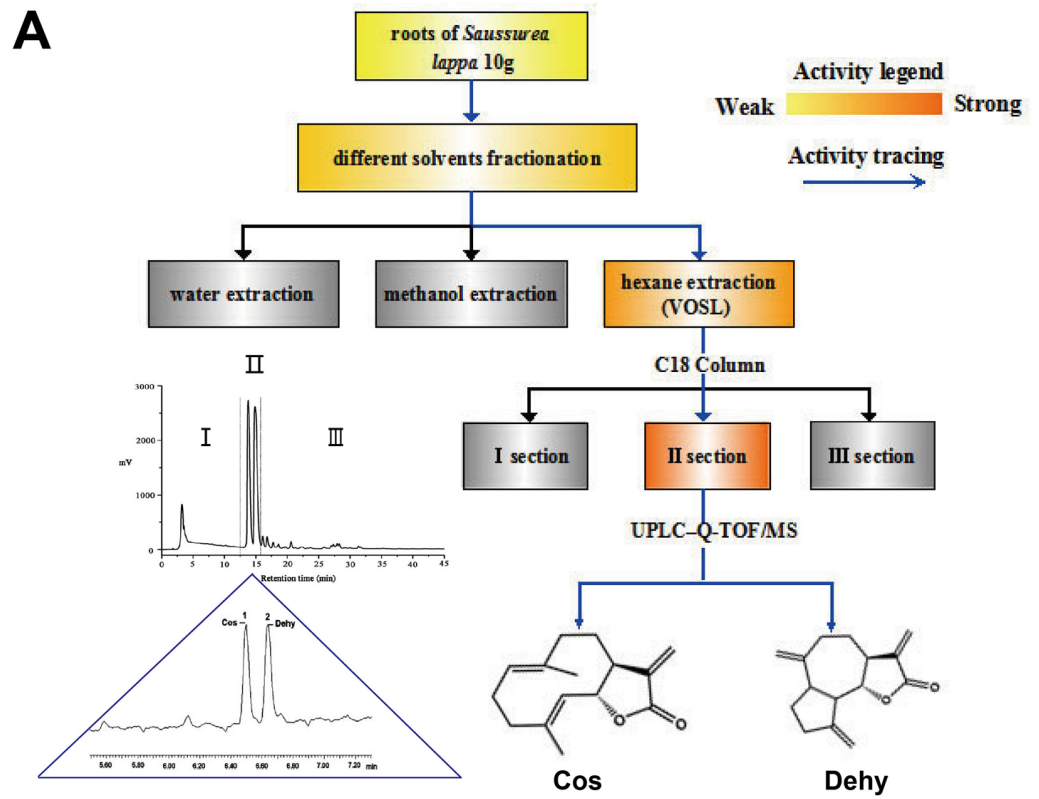

B

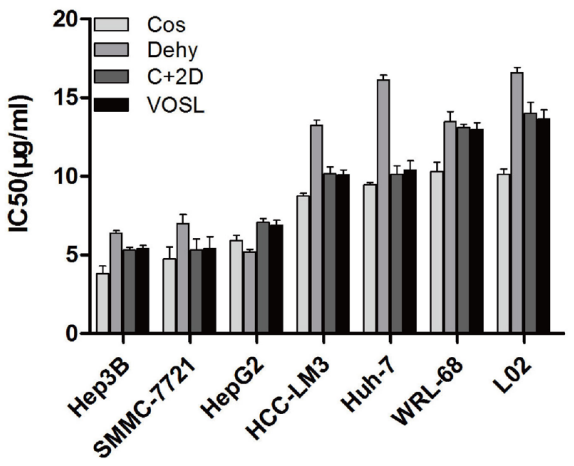

C



D

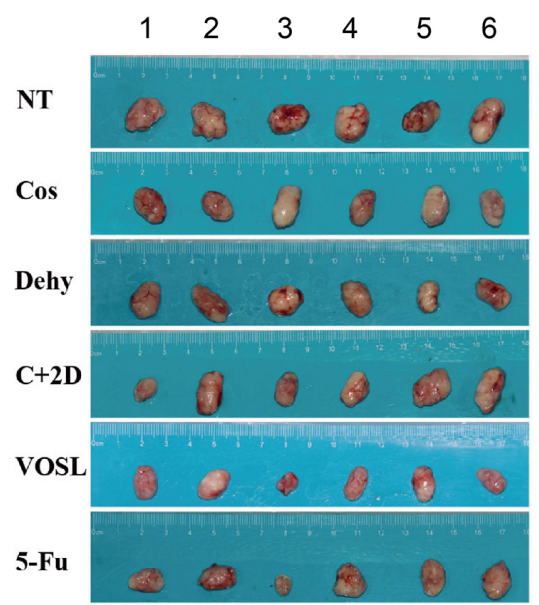

$\mathbf{E}$

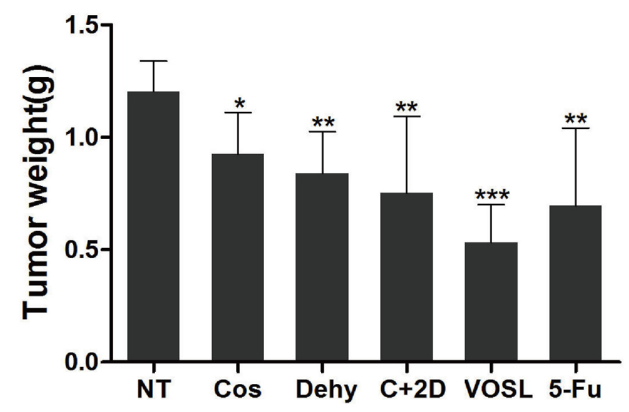

Figure 1: Therapeutic effects of VOSL and its main active ingredients on liver cancer. A. A flow diagram of VOSL isolation and identification of its active ingredients using a bioactivity-oriented screening platform. B. Cell lines were exposed to Cos, Dehy, C $+2 \mathrm{D}$ and VOSL for $48 \mathrm{~h}$. Cell viability was measured by MTT assay and the IC50 value of the tested samples was calculated using the Trimmed Spearman-Karber Method. C. SMMC-7721 cells were injected subcutaneously into the axillary fossa of nude mice until the tumors reached about 2-3 mm in diameter. The mice were treated with Cos, Dehy, C+2D, VOSL and 5-FU at the indicated concentrations, $\mathrm{n}=6$ in every group. Tumor volume was measured and compared; $* * p<0.01$ and $* * * p<0.001$ compared with the negative control (NT) group. D and E. The tumors were harvested and weighed after 24 times of administrations; ${ }^{*} p<0.05, * * p<0.01$ and $* * * p<0.001$ compared with the NT group. 
proliferation, survival or death of cancer cells [16]. Therefore, the effects of VOSL, C+2D, Cos, and Dehy on MAPK and Akt activation were examined. MEK/P38 cascade activation in SMMC-7721 and Hep3B cells was dose-dependently suppressed by VOSL, C+2D, Cos, and Dehy, but they had no effect on the activation of ERK. Meanwhile, VOSL, C+2D, and Dehy dose-dependently decreased p-Akt levels in SMMC-7721 and Hep3B cells, however, the p-Akt level had no obvious changes in the Cos-treated group (Figure 5A).

Since MEK/P38 and PI3-K/Akt is the primary downstream cascades of receptor tyrosine kinases (RTKs), meanwhile, EGFR is the most important RTK in cancer cells $[17,18]$. Therefore, we speculated that VOSL may act as an EGFR inhibitor to suppress EGFR activation. In order to validate this speculation, we determined the EGFR activation in the control group and the treatment groups. The results revealed that the phosphorylated EGFR (p-EGFR) levels in HCC cells were significantly suppressed after VOSL, C+2D, Cos, and Dehy treatments (Figure 5B).

\section{VOSL suppresses RTKs-mediated signaling in HCC xenografts}

The expression of relevant factors and the number of apoptotic cells in xenograft tumors were detected by immunohistochemistry and TUNEL assay, respectively. VOSL, C+2D, Cos, and Dehy treatments could induce SMMC-7721 cell apoptosis in xenograft tumors, among them, VOSL exerted the greatest effect on inducing cell apoptosis (Figure 6A and 6C). The phosphorylation of Akt was also blocked by VOSL, C+2D, and Dehy treatments in the SMMC-7721 xenografts, except the Cos-treated group (Figure 6A and 6B). Simultaneously, the expression of Ki67, p-P38 and p-EGFR in all treatment groups was reduced compared with the control group, and their lowest expression levels were observed in the VOSL-treated

A

B
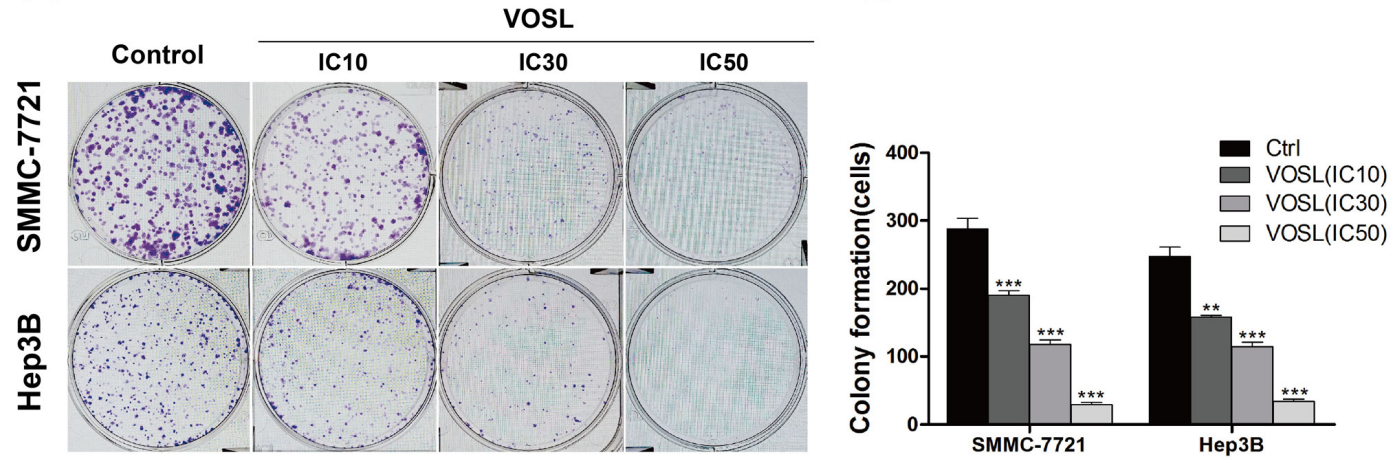

C
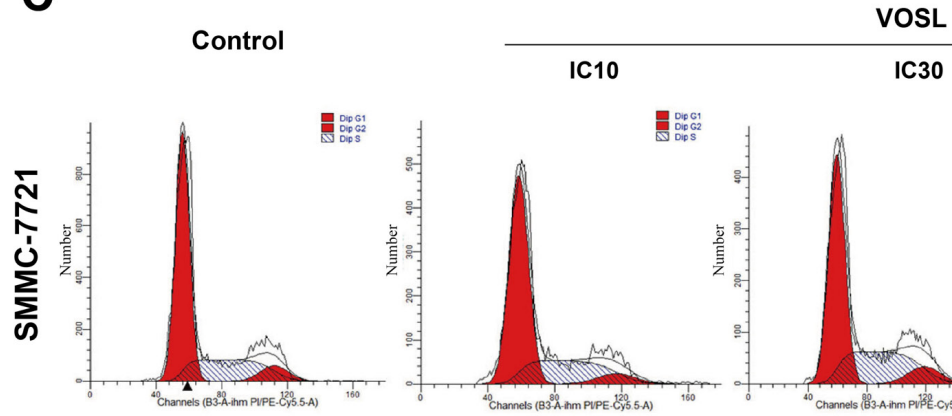

VOSL
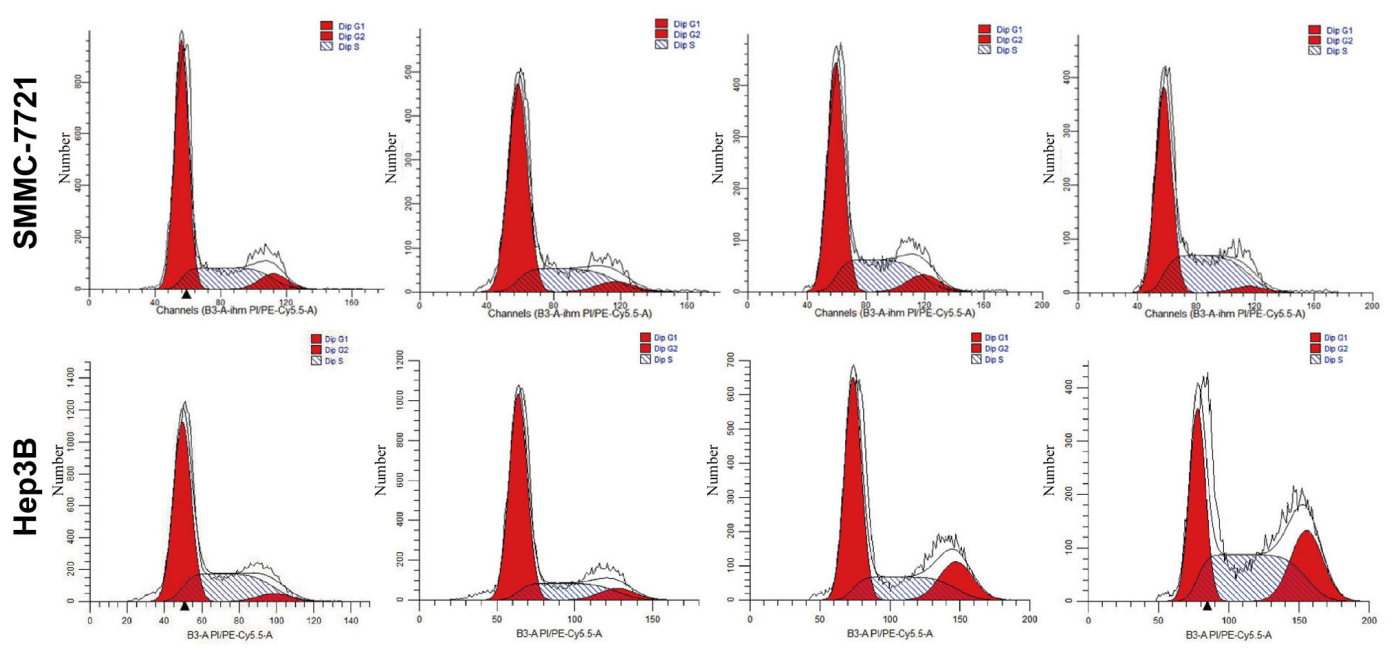

Figure 2: VOSL inhibits colony formation of $\mathrm{HCC}$ and influences the distribution of cell cycle. A and $\mathbf{B}$. Cells were treated with VOSL at different concentrations ( IC10, IC30, IC50) for $48 \mathrm{~h}$, and the colony formation was presented as mean \pm SD; $* * p<0.01$ and $* * * p<0.001$ compared with the control group. C. HCC cells were exposed to VOSL (IC10, IC30, IC50) for $48 \mathrm{~h}$ and cell cycle was detected by flow cytometry (Detail data shown in Table 1). 
Table 1: Cell cycle distribution after treatment of Cos, Dehy, C+2D or VOSL for 48 h

\begin{tabular}{|c|c|c|c|c|c|}
\hline Cell lines & Treatment & Concentration & G0/G1 (\%) & S (\%) & G2/M (\%) \\
\hline \multirow[t]{13}{*}{ SMMC-7721 } & Control & & $63.22 \pm 2.62$ & $28.57 \pm 1.97$ & $8.21 \pm 0.67$ \\
\hline & Cos & IC 10 & $59.43 \pm 0.62$ & $33.85 \pm 0.59 *$ & $6.72 \pm 1.00$ \\
\hline & & IC30 & $50.71 \pm 0.73 * *$ & $39.44 \pm 0.60 * *$ & $9.86 \pm 0.15$ \\
\hline & & IC50 & $41.77 \pm 0.30 * * *$ & $51.01 \pm 0.22 * * *$ & $7.22 \pm 0.33$ \\
\hline & Dehy & IC10 & $62.88 \pm 0.37$ & $30.84 \pm 0.61$ & $6.28 \pm 0.48$ \\
\hline & & IC30 & $55.57 \pm 0.83 * *$ & $35.89 \pm 0.66 *$ & $8.54 \pm 1.30$ \\
\hline & & IC50 & $49.44 \pm 0.90 *$ & $46.22 \pm 0.94 * * *$ & $4.34 \pm 1.18 * * *$ \\
\hline & $\mathrm{C}+2 \mathrm{D}$ & $\mathrm{IC} 10$ & $63.91 \pm 1.52$ & $31.25 \pm 0.89$ & $4.84 \pm 1.23 *$ \\
\hline & & IC30 & $53.26 \pm 0.39 * *$ & $37.86 \pm 0.94 * *$ & $8.88 \pm 0.84$ \\
\hline & & IC50 & $45.62 \pm 0.72 * * *$ & $48.57 \pm 0.45 * * *$ & $5.80 \pm 1.09 *$ \\
\hline & VOSL & IC10 & $62.67 \pm 1.55$ & $29.84 \pm 0.70$ & $7.42 \pm 0.96$ \\
\hline & & IC30 & $57.99 \pm 1.69 *$ & $34.09 \pm 0.29 * *$ & $7.91 \pm 1.96$ \\
\hline & & IC50 & $54.96 \pm 1.68 * *$ & $41.12 \pm 1.45 * * *$ & $3.92 \pm 0.46 *$ \\
\hline \multirow[t]{13}{*}{ Нер3B } & Control & & $67.44 \pm 1.35$ & $24.45 \pm 1.18$ & $8.11 \pm 0.30$ \\
\hline & $\mathrm{Cos}$ & IC10 & $65.85 \pm 3.14$ & $25.41 \pm 1.06$ & $8.74 \pm 3.29$ \\
\hline & & IC30 & $59.54 \pm 1.29 * *$ & $34.35 \pm 2.23 * *$ & $6.11 \pm 2.82$ \\
\hline & & IC50 & $49.71 \pm 2.09 * * *$ & $46.93 \pm 1.29 * * *$ & $3.36 \pm 2.05^{*}$ \\
\hline & Dehy & $\mathrm{IC} 10$ & $64.47 \pm 3.62$ & $26.14 \pm 0.86$ & $9.40 \pm 3.25$ \\
\hline & & IC30 & $60.16 \pm 2.34 * *$ & $36.01 \pm 2.16 * *$ & $3.83 \pm 2.92$ \\
\hline & & IC50 & $34.83 \pm 1.74 * * *$ & $46.90 \pm 1.08 * * *$ & $18.27 \pm 1.77 * * *$ \\
\hline & $\mathrm{C}+2 \mathrm{D}$ & $\mathrm{IC} 10$ & $64.88 \pm 2.14$ & $29.31 \pm 0.67 * *$ & $5.81 \pm 2.19$ \\
\hline & & IC 30 & $44.51 \pm 3.00 * * *$ & $36.22 \pm 0.95 * * *$ & $19.27 \pm 2.10 * * *$ \\
\hline & & IC50 & $32.72 \pm 0.82 * * *$ & $48.73 \pm 1.15 * * *$ & $18.54 \pm 1.46 * * *$ \\
\hline & VOSL & IC 10 & $53.43 \pm 0.74 * * *$ & $27.92 \pm 1.65 *$ & $18.65 \pm 1.03 * * *$ \\
\hline & & IC30 & $57.56 \pm 1.68 * *$ & $35.11 \pm 1.67 * * *$ & $7.34 \pm 1.63$ \\
\hline & & IC50 & $33.55 \pm 0.64 * * *$ & $43.78 \pm 1.25 * * *$ & $22.68 \pm 1.48 * * *$ \\
\hline
\end{tabular}

Note: $* p<0.05, * * p<0.01$ and $* * * p<0.001$ compared with the corresponding control group.

group (Figure 6A and 6B). Therefore, we concluded that VOSL may inhibit HCC progression through suppressing the RTKs-mediated signaling pathways.

\section{DISCUSSION}

$\mathrm{HCC}$ is one of the common malignances and has high mortality due to frequent recurrence, metastasis and multi-drug resistance $[19,20]$. Our study demonstrated that VOSL, C+2D, Cos and Dehy, acting as EGFR inhibitors, exhibited anti-HCC activity, and VOSL showed the best anti-HCC activity among the tested groups.

Unlimited proliferation is a hallmark of cancer cells, thus proliferation inhibition and apoptosis induction are the two main mechanisms of chemotherapeutic agents [21]. In this study, VOSL, C+2D, Cos and Dehy markedly inhibited the proliferation of SMMC-7721 and Hep3B cells, interfered the progression of cell cycle, and also triggered apoptosis to inhibit the progress of HCC. The apoptotic mechanisms in HCC cells induced by Cos may be partially different from those induced by VOSL, C+2D or Dehy due to the ratio of cleaved-Caspase 3 to Caspase3 was obviously increased in the VOSL, C+2D or Dehytreated groups, but it was no obvious change in the Costreated group. Activation of Caspase 3 is always involved in the regulation of intrinsic and extrinsic apoptotic pathways, and the activity of P38 has great relationship with the endoplasmic reticulum (ER) stress apoptotic 


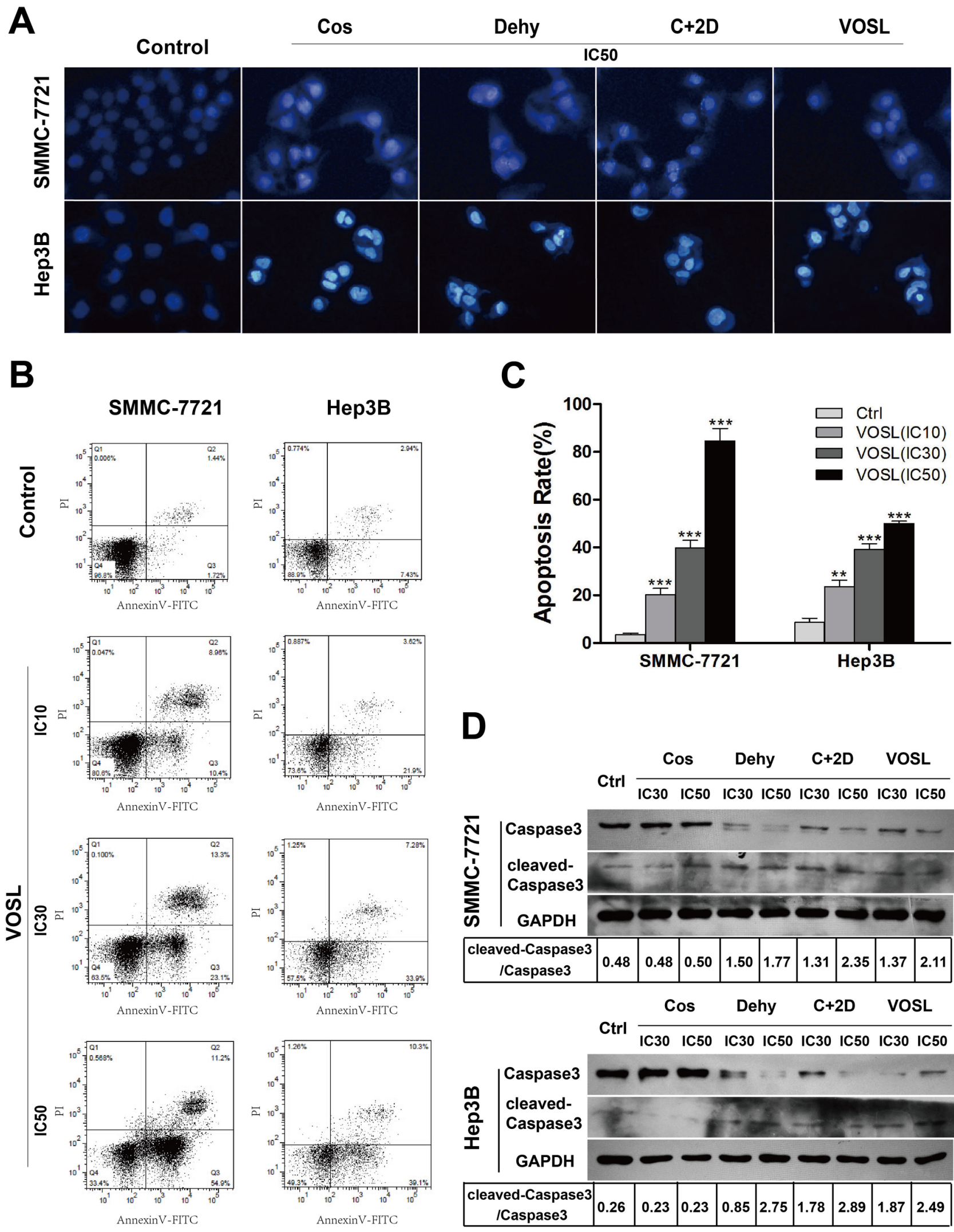

Figure 3: VOSL induces HCC cell apoptosis. A. Apoptotic changes induced by IC50 of Cos, Dehy, C+2D snd VOSL treatments for $48 \mathrm{~h}$ were observed by Hoechst 33342 staining in HCC cells. B and C. Apoptotic cells were determined by flow cytometry. The apoptotic percentages were presented as mean $\pm \mathrm{SD} ; * * p<0.01$ and $* * * p<0.001$ compared with the control group. D. The Caspase 3 and cleavedCaspase 3 levels in HCC cells were examined by Western blot after $48 \mathrm{~h}$ treatment. 
pathway [10]. In this study, we also found that the phosphorylation of P38 in SMMC-7721 and Hep3B cells was dose-dependently inhibited by VOSL, C+2D, Cos, and Dehy. Therefore, we suggested that VOSL, C+2D or Dehy-induced HCC cell apoptosis may adopts at least two

A

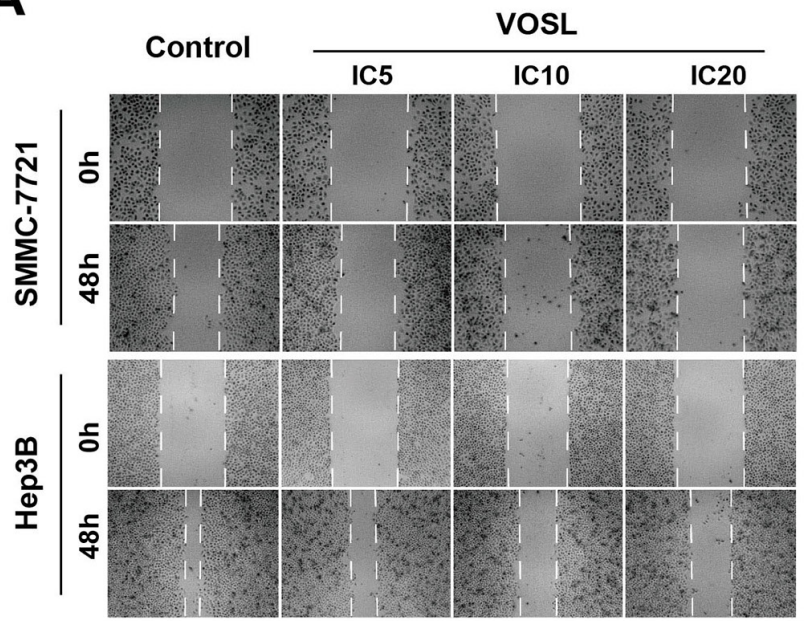

C

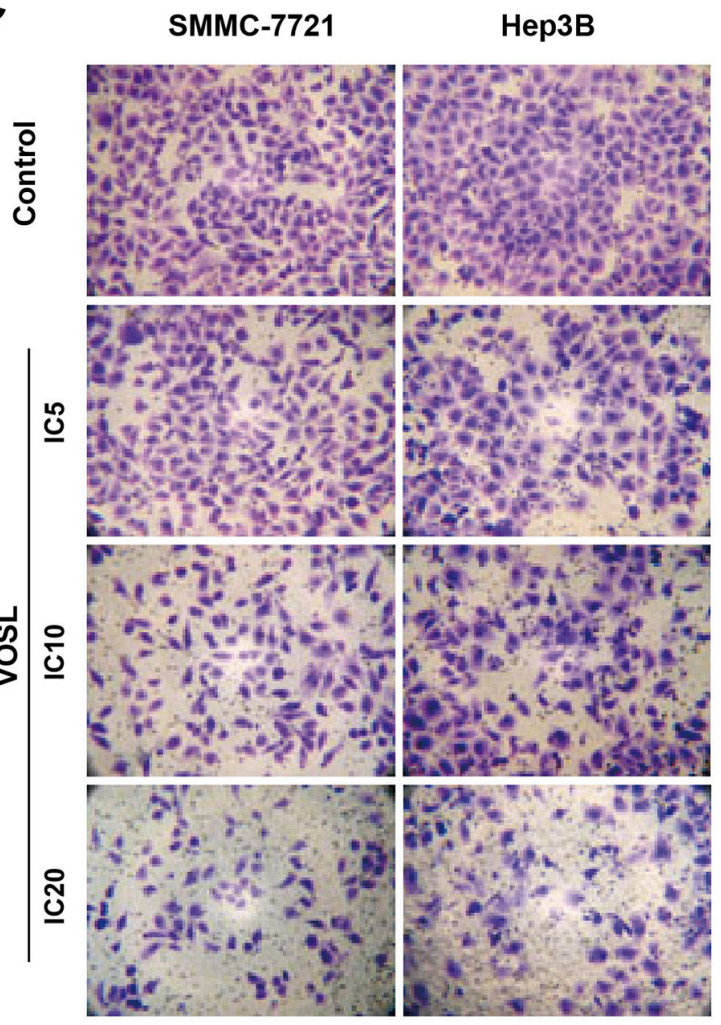

different apoptotic pathways, but Cos-induced HCC cell apoptosis possibly employs the ER stress pathway.

Numerous studies indicated that Cos and Dehy can modulate cell cycle progression by activating the P21/ P27 pathway, which lead to cell cycle arrest in S or/and

B

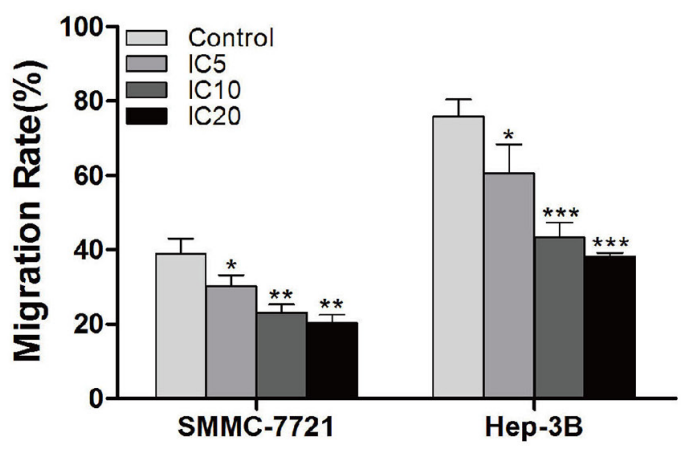

D

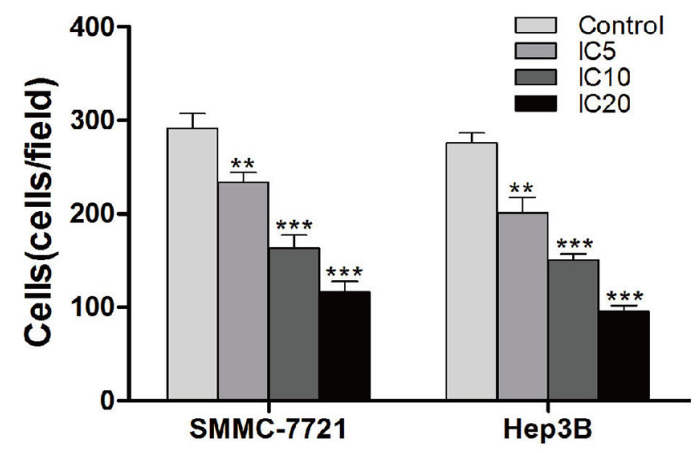

$\mathbf{E}$

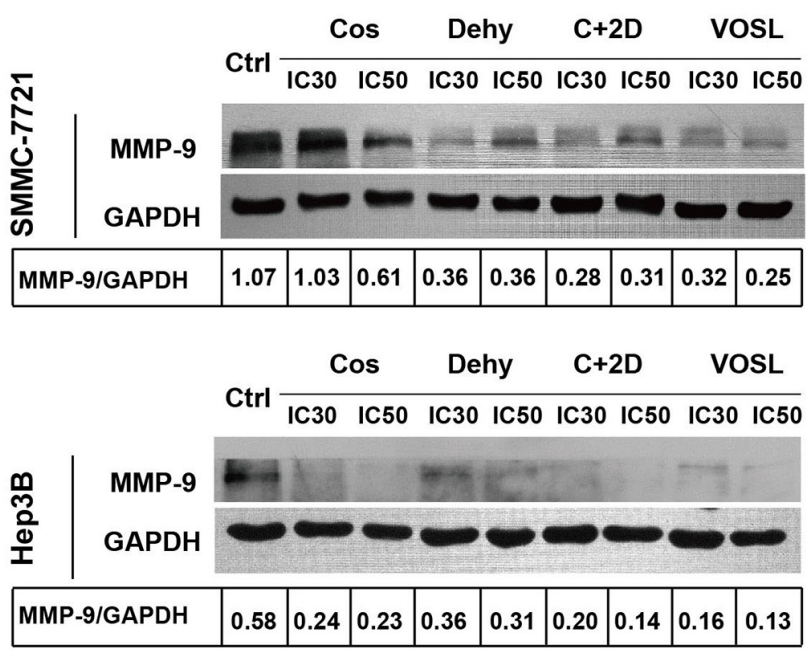

Figure 4: VOSL inhibits the motility activity of HCC cells. A and B. HCC cells were treated with VOSL (IC5, IC10, IC20) for $48 \mathrm{~h}$, and the effect of VOSL on cell migration was measured by scarification test. The migration rates of HCC cells were presented as mean $\pm \mathrm{SD} ;{ }^{*} p<0.05,{ }^{* *} p<0.01$ and ${ }^{* * *} p<0.001$ compared with the control group. C and D. After incubation with VOSL at IC5, IC10 and IC20 for $48 \mathrm{~h}$, the invasive property of HCC cells was tested in Transwell plates; original magnification 200×. The invasive cells were presented as mean $\pm \mathrm{SD} ; * *<<0.01$ and $* * * p<0.001$ compared with the control group. E. HCC cells were treated with Cos, Dehy, C+2D or VOSL (IC30, IC50) for $48 \mathrm{~h}$, and then the expression level of MMP-9 in HCC cells was examined by Western blot. 
G2/M phases $[22,23]$. In this study, we indeed observed that VOSL, C+2D, Dehy and Cos induce HCC cell cycle arrest in $\mathrm{S}$ or/and G2/M phases. MMP-9 can degrade some extracellular matrixs, and the change of MMP-9 expression is closely related to tumor metastasis and invasion in various tumors [24-28]. Our results from Scarification and Transwell assays indicated that VOSL, C+2D, Cos, and Dehy at low concentrations significantly reduced the capabilities of HCC cell migration and invasion. In addition, we observed the down-regulation of MMP-9 level in the VOSL, C+2D, Cos or Dehy- treated HCC cells.

Accumulating evidence showed that MAPK activation play a vital role in HCC development [17]. We found that VOSL, C+2D, Cos and Dehy treatments significantly depressed MEK/P38 cascade activation, but didn't influence ERK activation. PI3-K/Akt pathway is also involved in HCC progression. The activated Akt has been considered as an indicator for HCC recurrence and poor prognosis of patients [29]. Our results revealed that VOSL, C+2D, Cos and Dehy significantly suppressed the PI3-K/Akt signaling in HCC cells. Taken together, the simultaneous suppression of MEK/P38 and PI3-K/ Akt signaling always by VOSL represents an attractive strategy for treating HCC.

RTKs are glycoproteins spanning cell membrane, which interact with growth factors and in turn cause the activation of intracellular catalytic domains, whereby transmit cellular signals [30]. EGFR is the most important RTK in cancer cells and overexpresses in a variety of malignancies $[31,32]$. It can activate distinct downstream kinases, and leads to the phosphorylation of MAPK kinases and Akt [33-36]. Considering VOSL could simultaneously inhibit MEK/P38 and PI3-K/Akt signaling, we thought that VOSL may influence EGFR
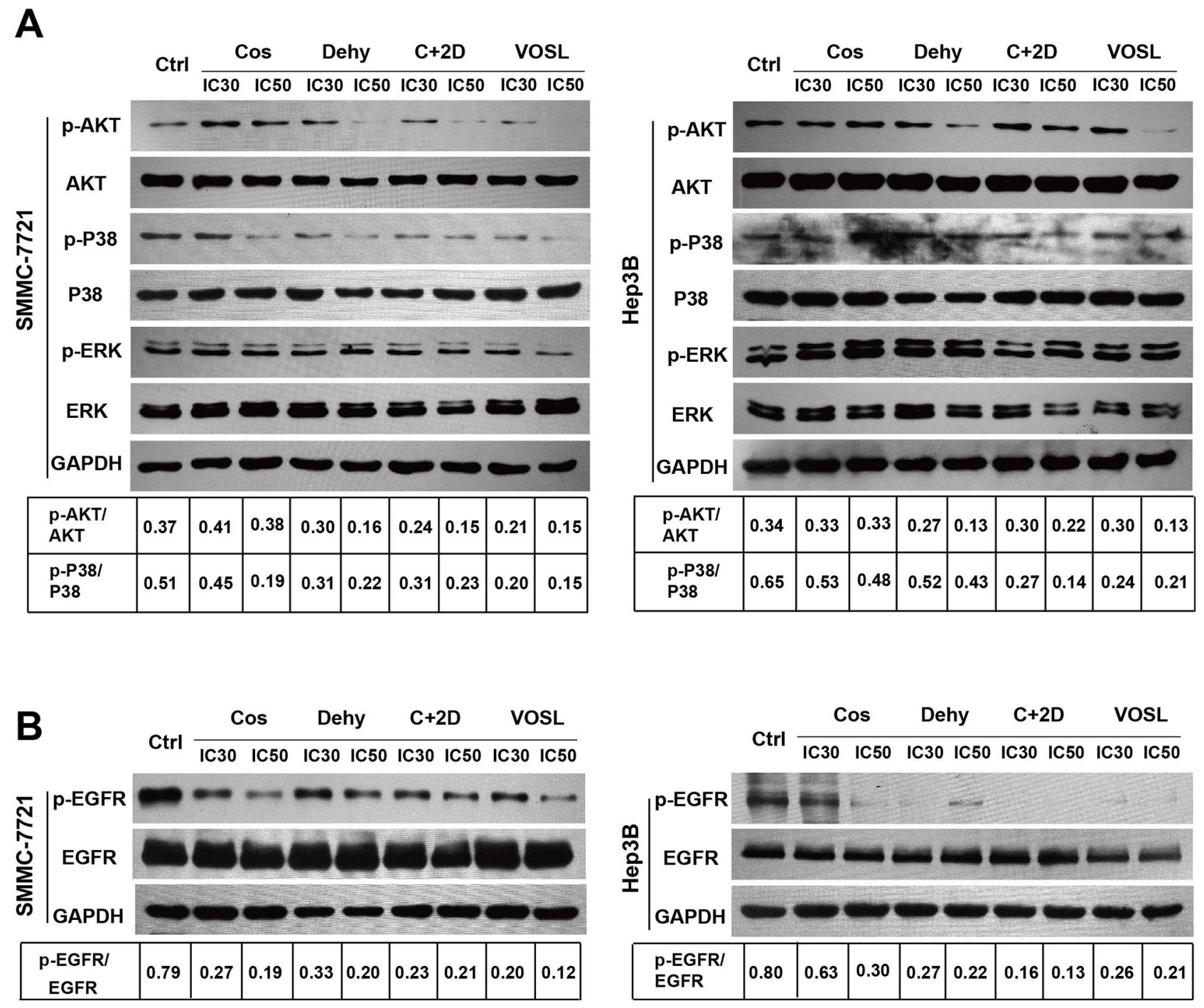

Figure 5: VOSL suppressed the EGFR/MEK/P38 and EGFR/PI3-K/Akt pathways in HCC cells. A and B. HCC cells were treated with Cos, Dehy, C+2D or VOSL at concentrations of 0, IC30 and IC50 for 48h, and then the expression levels of the relevant factors (P38, p-P38, Akt, p-Akt, ERK, p-ERK, EGFR and p-EGFR) were determined by Western blot. 
activation in HCC cells. Interestingly, our data showed that VOSL, C+2D, Cos and Dehy significantly suppressed the phosphorylation of EGFR in SMMC-7721 and Hep3B cells, which confirms our conjecture.

Although both Cos and Dehy are natural sesquiterpene lactones, their anti-HCC molecular mechanisms seem to be different. Combination treatment of these two compounds $(\mathrm{C}+2 \mathrm{D})$ showed a synergistic anti$\mathrm{HCC}$ effect in the SMMC-7721 xenografts. In addition, we speculated that there should be indirect anti-HCC compounds besides direct anti-HCC compounds, Cos and Dehy, in VOSL because VOSL exhibits better anti-HCC effect than $\mathrm{C}+2 \mathrm{D}$ in vivo. Therefore, VOSL exhibited the therapy characteristics of multiple-components and

A
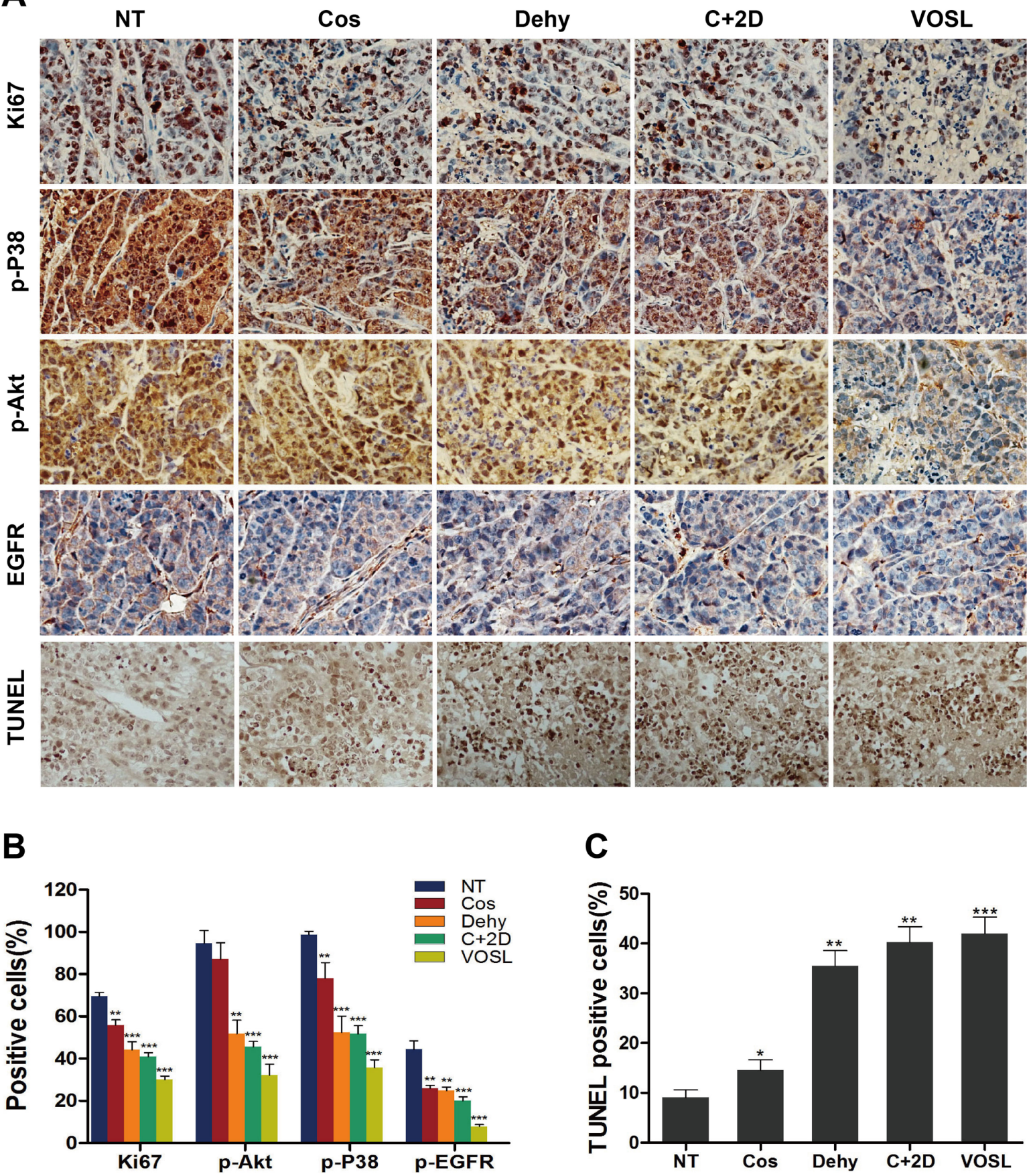

Figure 6: Expressions of the factors in SMMC-7721 xenografts after treatments. A. The expression of Ki-67, p-P38, p-Akt, p-EGFR and the apoptotic cells in xenografts were examined by immunohistochemistry and TUNEL assay; original magnification $200 \times$. $\mathbf{B}$ and $\mathbf{C}$. The positive cells of the relevant factors and the apoptotic cells in xenografts were presented as mean \pm SD; $* p<0.05, * * p<0.01$ and $* * * p<0.001$ compared with the NT control group. 
multiple-targets, which were Cos and Dehy acted on HCC xenografts directly, and other active ingredients in VOSL may act on nude mice to exert indirectly anti-HCC activity. Figuring out the indirect anti-HCC ingredients in VOSL and exploring their associated mechanism will be our topic of further research. In conclusion, our experiments showed that VOSL suppressed HCC progression by regulating EGFR pathway (Figure 7), which could be a useful drug candidate in $\mathrm{HCC}$ therapy.

\section{MATERIALS AND METHODS}

\section{Compounds}

VOSL was prepared as previously described [9]. Briefly, ten g of Saussurea lappa roots were crushed into powder, and extracted with $100 \mathrm{~mL}$ of hexane for $1 \mathrm{~h}$, The filtrates were evaporated to get VOSL, and then were analyzed by ultraperformance liquid chromatography coupled to quadrupole-time-of-flight mass spectrometer (Waters, Milford, MA, USA). Commercial Cos and Dehy were purchased from Shanghai Yuanye Biotech CO., Ltd and its purity is more than $98 \%$. VOSL, Cos and Dehy were dissolved in dimethyl sulfoxide to $10 \mathrm{mg} / \mathrm{mL}$, and then diluted to appropriate concentrations with cell culture medium.

\section{Cell culture}

Human HCC cell lines, SMMC-7721, Hep3B, HepG2, Huh-7, HCC-LM3, and two normal hepatic cell lines, WRL-68 and L02, were cultured in DMEM with $10 \%$ fetal bovine serum (HyClone, Logan, UT, USA), and incubated at $37^{\circ} \mathrm{C}, 5 \% \mathrm{CO}_{2}$.

\section{Cell proliferation}

The ratio of Cos to Dehy is about 1 to 2 by weight in VOSL, therefore, in the following experiments, we generally designed four tested groups, the Cos group, Dehy group, $\mathrm{C}+2 \mathrm{D}(\mathrm{Cos} / \mathrm{Dehy}=1: 2, \mathrm{w} / \mathrm{w}$; simulating the composition ratio of VOSL) group, and VOSL group, to treat HCC cells. Cell viability was detected by MTT assay. $0,5,10,15,20 \mu \mathrm{g} / \mathrm{ml}$ of the tested samples (Cos, Dehy, $\mathrm{C}+2 \mathrm{D}$ and VOSL) were added in cultured cells. After 48 $\mathrm{h}$ incubation, MTT reagent was added to each well, then

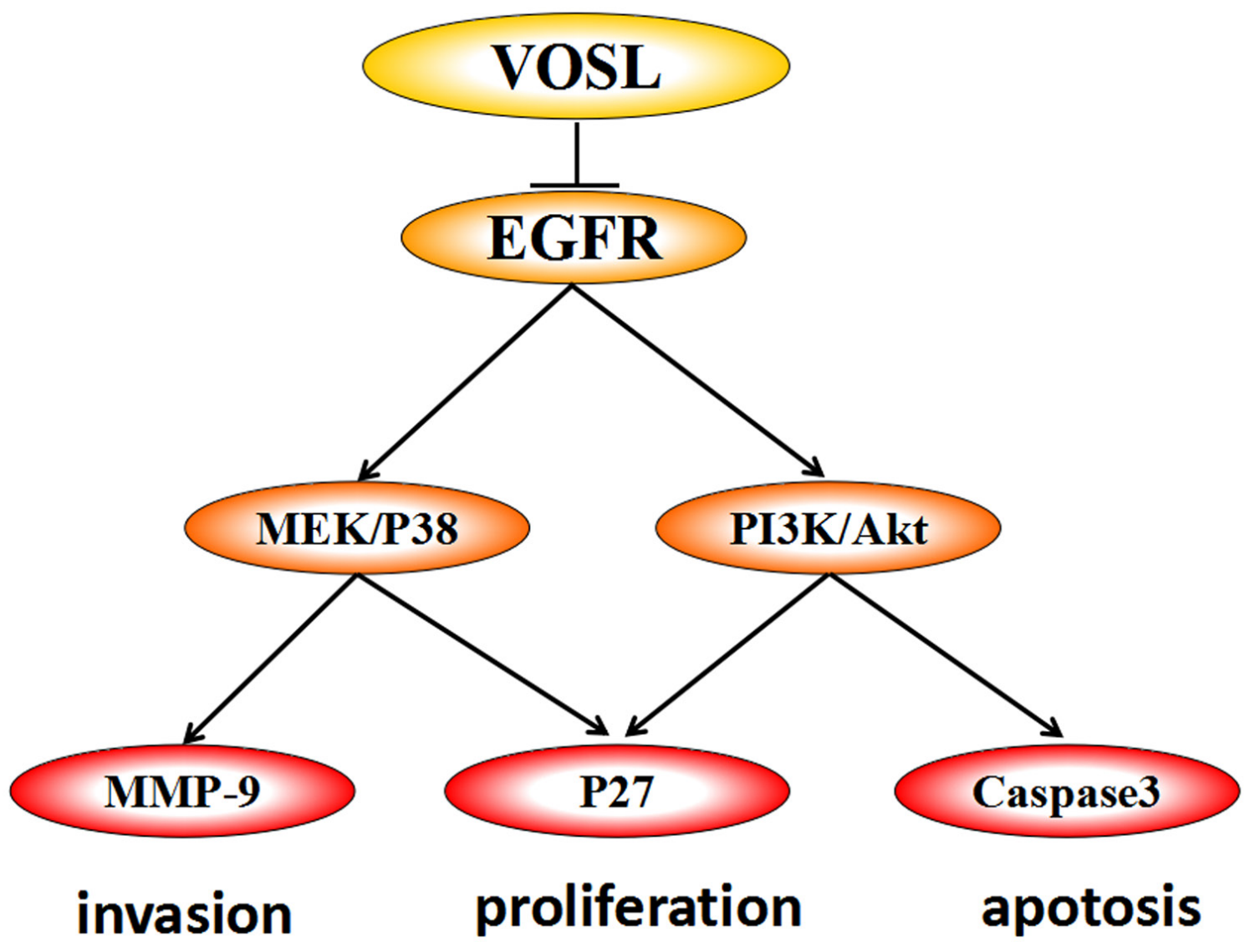

Figure 7: The model of how VOSL exerts antitumor efficacy in SMMC-7721 and Hep3B cells. VOSL exerts antitumor efficacy by inhibiting EGFR signaling pathway in hepatocellular carcinoma. The correlative pathways PI3K/Akt and MEK/P38 were suppressed, which resulted in downregulation of MMP-9 and Caspase3. 
the absorbanc at $492 \mathrm{~nm}$ was detected and the IC50 value of every tested sample was calculated using the Trimmed Spearman-Karber Method [13].

\section{Cell clonogenic and cell cycle assays}

To determine long-term effects of Cos, Dehy, $\mathrm{C}+2 \mathrm{D}$ or VOSL on the proliferation of SMMC-7721 and Hep3B cells, cells were exposed to the tested samples at IC10, IC30 and IC50 concentrations for $48 \mathrm{~h}$, respectively, and rinsed with fresh medium. The resulting adherent cells were cultured continuously for 14 days in normal culture condition. Finally, the cells were fixed and stained with crystal violet for counting the clone formation.

The distribution of cell cycle was detected by propidium iodid (PI) staining. SMMC-7721 and Hep3B cells were treated with Cos, Dehy, C+2D or VOSL at various concentrations (IC10, IC30 and IC50) for $48 \mathrm{~h}$. The harvested cells were fixed in alcohol (70\%) for $24 \mathrm{~h}$ at $4{ }^{\circ} \mathrm{C}$, stained with PI for $30 \mathrm{~min}$, then analyzed with flow cytometry (Becton Dickinson, Franklin Lakes, NJ, USA).

\section{Apoptosis analysis}

Hoechst was used to determine the morphological change of apoptotic cells. SMMC-7721 and Hep3B cells were treated with Cos, Dehy, C+2D or VOSL at IC50 concentration for $48 \mathrm{~h}$, and then stained with Hochest 33342 (Beyotime Biotechnology, Shanghai, China) for 15 min. A fluorescence microscope was used to observe the morphological change of apoptotic cells.

SMMC-7721 and Hep3B cells were treated with Cos, Dehy, C+2D or VOSL at IC10, IC30 and IC50 concentrations, respectively, for $48 \mathrm{~h}$, and stained with Annexin V-FITC/PI (Yesen, Biotechnology, Shanghai, China) for $10 \mathrm{~min}$, and then analyzed by FACS Calibur (Becton Dickinson, Franklin Lakes, NJ, USA) to calculated the apoptotic ratio of cells.

\section{Migration and invasion activity assays}

Scarification test was used to evaluate the migration of HCC cells. SMMC-7721 and Hep3B cells were planted in 6-well plates and wounds were created with a micropipette tip in the cell monolayer. The cells were exposed to Cos, Dehy, C+2D or VOSL at IC5, IC10, and IC20 concentrations, respectively, for $48 \mathrm{~h}$, and their migration distances were measured by Photoshop.

Transwell assay with extracellular matrix (ECM) was performed to evaluate the influence of Cos, Dehy, $\mathrm{C}+2 \mathrm{D}$ and VOSL on tumor invasion. SMMC-7721 and Hep3B were exposed to Cos, Dehy, C+2D or VOSL at IC5, IC10, and IC20 concentrations for $48 \mathrm{~h}$, and the infiltrated cells were stained with crystal violet and statistically analyzed by microscope.

\section{Protein extraction and western blot analysis}

SMMC-7721 and Hep3B cells were cultured and incubated with Cos, Dehy, C+2D or VOSL at IC30 and IC50 concentrations for $48 \mathrm{~h}$, respectively. The harvested cells were prepared to extract total protein. The extracted protein was separated on $8 \%-12 \%$ SDS-PAGE, then transferred onto PVDF membrane (Beyotime Institute of Biotechnology). The blot was blocked with non-fat milk, incubated with the primary antibodies (Caspase3, cleavedCaspase3, Akt, p-Akt, P38, p-P38, ERK, p-ERK, EGFR and p-EGFR) and then with the secondary antibodies. Signals were developed using ECL reagents (GE Healthcare Life Sciences, Shanghai, China). GAPDH was used as an inner control.

\section{In vivo tumor xenograft study}

All procedures involving animals were approved by the Experimental Animal Ethics and Management Committee. Male nude mice (4 weeks old; BALB/cnude) were purchased from SLAC Laboratory Animal Center of Chinese Academy of Sciences (Shanghai, China). SMMC-7721 cells were injected subcutaneously into the axillary fossa of nude mice $\left(4 \times 10^{6}\right.$ cells in 150 $\mu \mathrm{l}$ PBS). Tumors were developed at 10 days after cell injection. The treatment was started when tumors reached a size of $2-3 \mathrm{~mm}$ in diameter. Thirty-six mice were randomly divided into six groups. The Cos, Dehy, $\mathrm{C}+2 \mathrm{D}$ and VOSL-treated groups were injected intraperitoneally at a dose of $15 \mathrm{mg} / \mathrm{kg} / \mathrm{day}$, respectively. The positive and negative control group were administrated with 5 -Fluorouracil (5-Fu) at a dose of $15 \mathrm{mg} / \mathrm{kg} / \mathrm{day}$, and an equal volume of vehicle, respectively. Tumor size was monitored every three days, and tumor volume was calculated using the formula: tumor volume $=0.5 \times a \times b^{2}$, where $a$ and $b$ represent the largest and smaller diameters, respectively.

Tumor-bearing mice were sacrificed after 24 times of administrations. Tumors were collected and weighed, and then prepared the consecutive sections for examining the expression of p-AKT, p-P38, Ki-67 and p-EGFR by immunohistochemistry. The apoptotic cells in xenograft tumors were detected by TUNEL (Beyotime Institute of Biotechnology, Shanghai, China). The positive cells were observed under microscope within 5 medium-power fields at magnification $200 \times$.

\section{Statistical analysis}

The in vitro experimental data were derived from at least three independent experiments, and the in vivo data were collected from 6 mice. All data were expressed as mean $\pm \mathrm{SD}$, and the significant difference was determined by using the Student's $t$-test. The statistical significance was set at $p<0.05$. 


\section{ACKNOWLEDGMENTS}

This work was supported by the National Natural Science Foundation of China (81370552 and 81572863 to C. Su, 81503307 to Z. Peng, 81402565 to W. Ji, and 81500470 to $\mathrm{Y}$. Xu).

\section{CONFLICTS OF INTEREST}

The authors declare no conflicts of interest.

\section{REFERENCES}

1. Laursen L. A preventable cancer. Nature. 2014; 516:S2-S3.

2. Kojiro M. Histopathology of liver cancers. Best Pract Res Cl Ga. 2005; 19:39-62.

3. Fattovich G, Stroffolini T, Zagni I, Donato F. Hepatocellular carcinoma in cirrhosis: incidence and risk factors. Gastroenterology. 2004; 127:S35-50.

4. Mazzaferro V, Chun YS, Poon RT, Schwartz ME, Yao FY, Marsh JW, Bhoori S, Lee SG. Liver transplantation for hepatocellular carcinoma. Ann Surg Oncol. 2008; 15:1001-1007.

5. Llovet JM, Bruix J. Systematic review of randomized trials for unresectable hepatocellular carcinoma: Chemoembolization improves survival. Hepatology. 2003; 37:429-442.

6. Bindseil KU, Jakupovic J, Wolf D, Lavayre J, Leboul J, van der Pyl D. Pure compound libraries: a new perspective for natural product based drug discovery. Drug Discovery Today. 2001; 6:840-847.

7. Firn RD, Jones CG. Natural products-a simple model to explain chemical diversity. Nat Pro Rep. 2003; 20:382-391.

8. Pan L, Chai H, Kinghorn AD. The continuing search for antitumor agents from higher plants Phytochem Lett. 2010; $12 ; 1-8$.

9. Peng ZX, Wang Y, Gu X, Wen YY, Yan C. A platform for fast screening potential anti-breast cancer compounds in traditional Chinese medicines. Biomed Chromatogr. 2013; 27:1759-1766.

10. Lin X, Peng Z, Su C. Potential Anti-Cancer Activities and Mechanisms of Costunolide and Dehydrocostuslactone. Int J Mol Sci. 2015; 16:10888-10906.

11. Chen HC, Chou CK, Lee SD, Wang JC, Yeh SF. Active compounds from Saussurea lappa Clarks that suppress hepatitis B virus surface antigen gene expression in human hepatoma cells. Antivir Res. 1995; 27:99-109.

12. Peng ZX, Wang Y, Gu X, Xue Y, Wu Q, Zhou J, Chan Y. Metabolic transformation of breast cancer in a MCF-7 xenograft mouse model and inhibitory effect of volatile oil from Saussurea lappa Decne treatment. Metabolomics. 2014; 11:636-656.

13. Hamilton MA, Russo RC, Thurston RV. Trimmed Spearman-Karber Method for estimating median lethal concentrations in toxicity bioassays. Environ Sci Technol. 1978; 12:714-719.

14. Koul HK, Pal M, Koul S. Role of p38 MAP Kinase Signal Transduction in Solid Tumors. Genes Cancer. 2013; 4:342359. doi: 10.1177/1947601913507951.

15. Langlois B, Perrot G, Schneider C, Henriet P, Emonard H, Martiny L, Dedieu S. LRP-1 Promotes Cancer Cell Invasion by Supporting ERK and Inhibiting JNK Signaling Pathways. PLoS One. 2010; 5:e11584.

16. Chiang EP, Tsai SY, Kuo YH, Pai MH, Chiu HL, Rodriguez RL, Tang FY. Caffeic Acid Derivatives Inhibit the Growth of Colon Cancer: Involvement of the PI3-K/Akt and AMPK Signaling Pathways. PLoS One. 2014; 9:e99631.

17. Wang SQ, Liu ST, Zhao BX, Yang FH, Wang YT, Liang QY, Sun YB, Liu Y, Song ZH, Cai Y, Li GF. Afatinib reverses multidrug resistance in ovarian cancer via dually inhibiting ATP binding cassette subfamily B member 1 . Oncotarget. 2015; 6:26142-26160. doi: 10.18632/oncotarget.4536.

18. Diez-Cecilia E, Carson R, Kelly B, van Schaeybroeck S, Murray JT, Rozas I. Probing a 3,4'-bis-guanidinium diaryl derivative as an allosteric inhibitor of the Ras pathway. Bioorg Med Chem Lett. 2015; 25:4287-4292.

19. Wu Q, Wang R, Yang Q, Hou X, Chen S, Hou Y, Chen C, Yang Y, Miele L, Sarkar FH, Chen Y, Wang Z. Chemoresistance to gemcitabine in hepatoma cells induces epithelial-mesenchymal transition and involves activation of PDGF-D pathway. Oncotarget. 2013; 4:1999-2009. doi: 10.18632/oncotarget.1471.

20. Guan DX, Shi J, Zhang Y, Zhao JS, Long LY, Chen TW, Zhang EB, Feng YY, Bao WD, Deng YZ, Qiu L, Zhang XL, Koeffler HP, et al. Sorafenib enriches epithelial cell adhesion molecule-positive tumor initiating cells and exacerbates a subtype of hepatocellular carcinoma through TSC2-AKT cascade. Hepatology. 2015; 62:1791-803.

21. Ding J, Wen W, Xiang D, Yin P, Liu Y, Liu C, He G, Cheng Z, Yin J, Sheng C, Zhang W, Nan F, Ye W, et al. $\psi$-Bufarenogin, a novel anti-tumor compound, suppresses liver cancer growth by inhibiting receptor tyrosine kinasemediated signaling. Oncotarget. 2015; 6:11627-11639. doi: 10.18632/oncotarget.3435.

22. Noh HJ, Kim KA, Kim KC. p53 down-regulates SETDB1 gene expression during paclitaxel induced-cell death. Biochem Bioph Res Co. 2014; 446:43-48.

23. Gismondi A, Canuti L, Impei S, di Marco G, Kenzo M, Colizzi V, Canini A. Antioxidantextracts of African medicinal plants induce cell cycle arrest and differentiation in B16F10 melanoma cells. Int J Oncol. 2013; 43:956-964.

24. Mehlen P, Puisieux A. Metastasis: A question of life or death. Nat Rev. 2006; 6:449-458.

25. Kessenbrock K, Plaks V, Werb Z. Matrix metalloproteinases: Regulators of the tumor microenvironment. Cell. 2010; 141:52-67.

26. Bourboulia D, Stetler-Stevenson WG. Matrix metalloproteinases (MMPs) and tissue inhibitors of 
metalloproteinases (TIMPs): Positive and negative regulators in tumor cell adhesion. Semin. Cancer Biol. 2010; 20:161-168.

27. Roy R, Yang J, Moses MA. Matrix metalloproteinases as novel biomarkers and potential therapeutic targets in human cancer. J Clin Oncol. 2009; 27:5287-5297.

28. Stamenkovic I. Matrix metalloproteinases in tumor invasion and metastasis. Semin Cancer Biol. 2000; 10:415-433.

29. Wen W, Ding J, Sun W, Fu J, Chen Y, Wu K, Ning B, Han T, Huang L, Chen C, Xie D, Li Z, Feng G, et al. CyclinG1-mediated epithelial-mesenchymal transition via phosphoinositide 3-kinase/Akt signaling facilitates liver cancerprogression. Hepatology. 2012; 55:1787-1798.

30. Gur S, Sikka SC, Abdel-Mageed AB, Abd Elmageed ZY, Rezk B, Pankey E, Kadowitz PJ, Hellstrom WJ. Imatinib Mesylate (Gleevec) Induces Human Corpus Cavernosum Relaxation by Inhibiting Receptor Tyrosine Kinases (RTKs): Identification of New RTK Targets. Urology. 2013; 82:e11-6.

31. Acunzo M, Romano G, Palmieri D, Lagana A, Garofalo M, Balatti V, Drusco A, Chiariello M, Nana-Sinkam P, Croce
CM. Cross-talk between MET and EGFR in non-small cell lung cancer involves miR-27a and Sprouty2. Proc Nat. Acad Sci USA. 2013; 110:8573-8578.

32. Puri N, Salgia R. Synergism of EGFR and c-Met pathways, cross-talk and inhibition, in non-small cell lung cancer. $J$ Carcinog. 2008; 7:9.

33. Turjanski AG, Vaque JP, Gutkind JS. MAP kinases and the control of nuclear events. Oncogene. 2007; 26:3240-3253.

34. Marshall J. Clinical implications of the mechanism of epidermal growth factor receptor inhibitors. Cancer. 2006; 107:1207-1218.

35. Tomas A, Futter CE, Eden ER. EGF receptor trafficking: consequences for signaling and cancer. Trends Cell Biol. 2014; 24:26-34.

36. Mlcochova J, Faltejskova P, Nemecek R, Svoboda M, Slaby O. MicroRNAs targeting EGFR signalling pathway in colorectal cancer. J Cancer Res Clin. 2013; 139:1615-1624. 